\title{
Article \\ Pain Relief after Extracorporeal Shock Wave Therapy for Patellar Tendinopathy: An Ultrasound Evaluation of Morphology and Blood Flow
}

\author{
Toshihiro Maemichi ${ }^{1, *(D)}$, Toshiharu Tsutsui ${ }^{1}$, Takumi Okunuki ${ }^{1}$, Takuma Hoshiba ${ }^{2}$ and Tsukasa Kumai ${ }^{3, *(D)}$ \\ 1 Graduate School of Sport Sciences, Waseda University, 2-579-15 Mikajima, \\ Tokorozawa 359-1192, Saitama, Japan; t-t_y-g_1798@fuji.waseda.jp (T.T.); t.okunuki@akane.waseda.jp (T.O.) \\ 2 Institute for Sports Science, Waseda University, 2-579-15 Mikajima, Tokorozawa 359-1192, Saitama, Japan; \\ quma2012@gmail.com \\ 3 Faculty of Sport Sciences, Waseda University, 2-579-15 Mikajima, Tokorozawa 359-1192, Saitama, Japan \\ * Correspondence: t.m.waseda@ruri.waseda.jp (T.M.); kumakumat@waseda.jp (T.K.)
}

Citation: Maemichi, T.; Tsutsui, T.; Okunuki, T.; Hoshiba, T.; Kumai, T. Pain Relief after Extracorporeal Shock Wave Therapy for Patellar Tendinopathy: An Ultrasound Evaluation of Morphology and Blood Flow. Appl. Sci. 2021, 11, 8748. https://doi.org/10.3390/app11188748

Academic Editor: Alessandro de Sire

Received: 15 August 2021

Accepted: 17 September 2021

Published: 20 September 2021

Publisher's Note: MDPI stays neutral with regard to jurisdictional claims in published maps and institutional affiliations.

Copyright: (c) 2021 by the authors. Licensee MDPI, Basel, Switzerland. This article is an open access article distributed under the terms and conditions of the Creative Commons Attribution (CC BY) license (https:/ / creativecommons.org/licenses/by/ $4.0 /)$.

\begin{abstract}
We aimed to investigate the changes caused by focused extracorporeal shock wave pain therapy (f-ESWT) in patients with patellar tendinopathy by means of ultrasound imaging. We included 18 knees from 11 college athletes with patellar tendinopathy. We assessed the tendon thickness and blood flow of the patellar tendon using ultrasound imaging, rest pain using NRS and tenderness using a pressure pain gauge. We recorded four measurements: immediately before f-ESWT (PRE(1)) and after f-ESWT (POST(1)) and two weeks after the first irradiation before f-ESWT (PRE(2) and after f-ESWT (POST(2)). Only the resting pain in both the first and second irradiations showed a significant difference immediately before and after the treatment. In terms of pain changes after two weeks later, we observed significant differences in the resting pain between PRE(1) and PRE(2) and also in the resting pain, tenderness and blood flow area between PRE(1) and POST(2). No significant difference was seen in the tendon thickness. We concluded that pain in the patellar tendon at rest decreased before and after irradiation, suggesting that f-ESWT may have influenced the nociceptive structures and had an analgesic effect.
\end{abstract}

Keywords: patellar tendinopathy; focus extracorporeal shock wave therapy; ultrasound; imaging analysis; blood flow; sports; athlete; overuse; treatment; morphology

\section{Introduction}

Focused extracorporeal shock wave pain therapy (f-ESWT), one of the pain treatment methods for tendinopathies, has received increasing attention in recent years. f-ESWT's main effects are pain relief [1], tissue repair [2] and bone formation promotion [3]. In addition, many efficacy studies on tendon disorders have been reported [3-6]. Because of its low complications and high safety, f-ESWT is gaining popularity as a treatment that should be considered before surgery, making it an attractive and safe option for athletes aiming for an early return to their sports.

Patellar tendinopathy is an overuse disorder frequently seen in sportspersons who participate in jumping, dashing and turning motions, such as in volleyball $[7,8]$ and basketball $[9,10]$. The patellar tendon connects the patella to the tibia and plays a role in knee flexion and extension by transmitting the tension of the quadriceps muscle. Therefore, excessive tension in the patellar tendon due to jumping and dashing motions leads to microdamage and inflammation of the tendon's parenchyma, thereby resulting in patellar tendinopathy $[11,12]$. The main pathogenesis of patellar tendinopathy includes degeneration and collagen malalignment, collagen changes from type I to Type III of the tendon, abnormal blood vessels in the sparing area and increased growth of nerve fibres $[10,13,14]$. It also inhibits tissue repair due to the excessive localisation of neurotransmitters [15]. 
Conservative therapy, including eccentric exercise, is the main treatment for patellar tendinopathy. However, over a long period, competitive athletes often develop intractable patellar tendinopathy with prolonged pain [16], and in some cases, surgery is required because conservative therapy is not successful.

In addition, the change in tendon stiffness due to scarring reduces its ability to transmit tension, making patellar tendinopathy directly related to decreased athletic performance [17]. Intractable patellar tendinopathy is a serious condition that can cause the end of a career for athletes because surgical treatment requires prolonged withdrawal from sporting competitions.

The efficacy of f-ESWT for patellar tendinopathy is $60-90 \%$, but the mechanism of healing is unclear [18-20]. A few reports deny the efficacy of f-ESWT and suggest that the results of f-ESWT are poor in refractory patellar tendinopathy and tendinopathy with advanced lesions. Objectively evaluating the actual patellar tendinopathy site, rather than carrying out only subjective pain assessments, may lead to further clarification of the cause of tendinopathy [21-24]. Ultrasound echo can be used not only to gain a more detailed picture of tendon morphology but also to map changes in tendon blood flow components in detail. In addition, recent developments in ultrasound echo have made it possible to capture the internal structure in detail before, making it possible to clearly see Doppler images. By using these, it can be used as an objective index to evaluate the effect of f-ESWT in more detail. Therefore, in this study, we examined the effects of f-ESWT on immediate and short-term pain changes by performing ultrasound imaging of the patellar tendon in patients with patellar tendinopathy.

\section{Materials and Methods}

\subsection{Participants}

We included 18 knees (male: 4, female: 7) from 11 university athletes belonging to university sports clubs who were examined by a physician at a university medical science clinic and diagnosed with patellar tendinopathy from June 2018 to June 2019 (age: $20.6 \pm 1.6$ years, height: $171.3 \pm 10.9 \mathrm{~cm}$, weight: $69.7 \pm 18.2 \mathrm{~kg}, \mathrm{BMI}: 23.5 \pm 4.2 \mathrm{~kg} / \mathrm{m}^{2}$, duration of symptoms: $19.5 \pm 11.3$ months). The sports that the subjects were involved in were soccer for 3 , American football for 3, long-distance runners for 2, basketball for 1 and volleyball for 2 .

All the subjects had patellar tendinopathy of an intensity that interfered with their sports activities or daily life, i.e., they had stage 3 or higher of patellar tendinopathy severity as defined by Blazina et al. [25-29] (Stage0; No pain, Stage1; Pain only after intense sports activity: no undue functional impairment, Stage2; Pain at the beginning and after sports activity: still able to perform at a satisfactory level, Stage3; Pain during sports activity: increasing difficulty in performing at a satisfactory level, Stage4; Pain during sports activity: unable to participate in sports at a satisfactory level, Stage5; Pain during sports activity: unable to participate in sports at a satisfactory level). We did not restrict them from any sports activities during the f-ESWT application period. This study was approved by the Ethical Review Committee for Research Involving Human Subjects (2017-318). The purpose of this study was fully explained to the participants, and consent was obtained before the study was conducted.

\subsection{Protocol}

We used an extracorporeal shock wave pain treatment device (STORZ Medical, UK) to deliver the f-ESWT to the pain-inducing area. We gradually increased the f-ESWT energy transfer in the range of $0.01-0.25 \mathrm{~mJ} / \mathrm{mm}^{2}$ according to the pain, delivering 2500 shots per session for $4 \mathrm{~Hz}$. The subjects were irradiated with f-ESWT twice, at the beginning of the study and after 2 weeks. We evaluated the pain, rest pain and tenderness of the patellar tendon. Rest pain was mapped on the Numeric Rating Scale (NRS) [30] and the degree of pain was rated on 11 levels. We then used a pressure pain meter (FP meter, SN-402, Navis, Japan) to evaluate the pressure pain and recorded the pressure value at 
the time the participant experienced pain [31-33]. When the pressure pain meter was applied to the pain area and pushed in, the value increased from 0 to $10(\mathrm{~kg})$. The examiner pushed it in until the study participant felt pain and recorded the value at the time of pain as the pressure value. In other words, it was shown that the higher pressure value indicated a decrease in pain. To examine the effect of f-ESWT on the affected area, we evaluated the tendon thickness and blood flow of the patellar tendon using an ultrasound imaging system, Toshiba Aplio scanning machine (Canon Medical Systems Corporation, Japan). Ultrasound evaluations of the tendon thickness and blood flow were performed in the long axis direction using an ultrasonography device equipped with a high-frequency (5-14 MHz) linear probe. To measure the tendon thickness $(\mathrm{mm})$, we recorded a still image of the area of maximum swelling using B-mode (Figure 1). For blood flow evaluation (Doppler), we recorded still images during the beating phase when the blood flow area was large $\left(\mathrm{cm}^{2}\right)$. Each image was captured by an orthopaedic surgeon who examined the subject and selected the images to be used. Four sessions in total were taken for evaluation by the ultrasound imaging system before and after the f-ESWT in each subject. Three images of tendon thickness and blood flow evaluation were recorded in each imaging session, and the average of the 3 images was adopted as the value. These recorded images were used to calculate the blood flow area on the ultrasound image using the open-access image analysis software Fiji (Fiji is Just ImageJ) (Figure 2). The shock wave irradiation site, pain evaluation site and ultrasound evaluation site were evaluated and determined by a physician using an ultrasound imaging device, and markings were applied thus that measurements could be performed at the same site. The measurements were performed 4 times: before f-ESWT (PRE(1), immediately after f-ESWT (POST(1)), before f-ESWT 2 weeks after the first f-ESWT (PRE(2) and immediately after the second f-ESWT (POST(2).

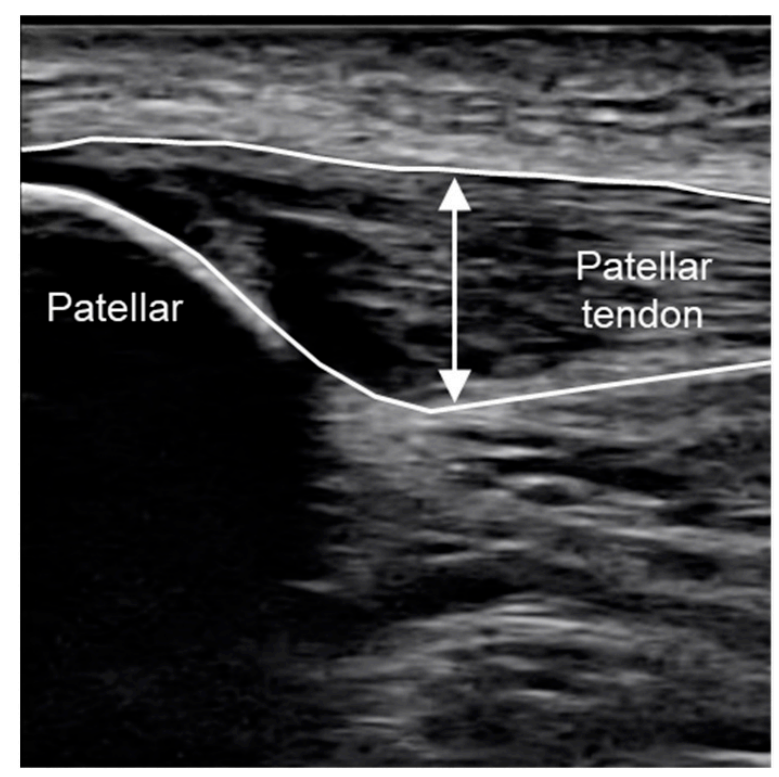

Figure 1. Measurement for Patellar tendon thickness.

\subsection{Statistical Analysis}

All data obtained in this study were expressed as mean \pm standard deviation. SPSS Statistics 26 (IBM, USA) was used for statistical analysis. Each measurement item was analysed using a one-way analysis of variance, and when the main effect was observed, multiple comparisons using Tukey's test were conducted as a post-test. The significance level was set at a risk rate of less than $5 \%$. 

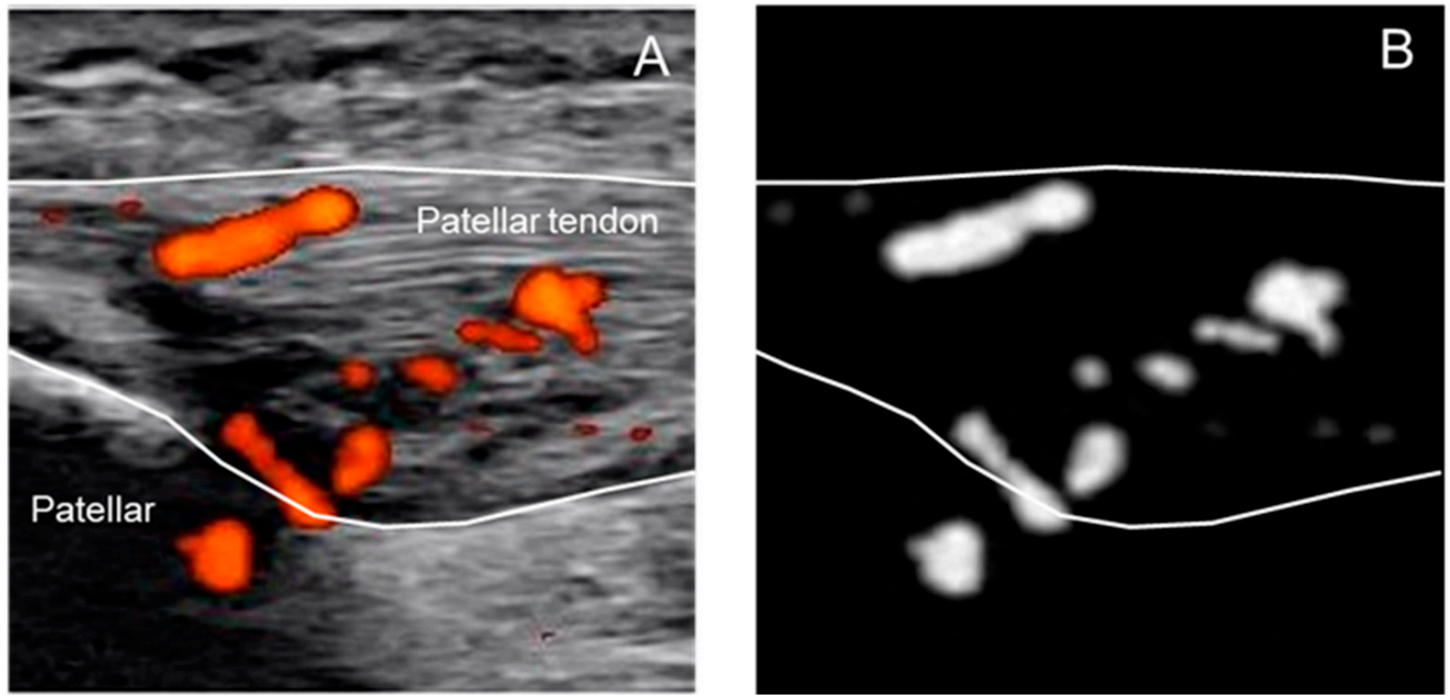

Figure 2. Measurement for blood flow area, (A) ultrasound Image, (B) analysis image using image J.

\section{Results}

The results of all evaluation items are shown in Table 1. In the immediate pain change before and after irradiation, we found a significant difference only in NRS after the first and second irradiations and no significant difference in the other items. In terms of pain change after 2 weeks, there was a significant difference in NRS between PRE(1) and PRE(2), and between PRE(1) and POST(2) (Figure 3). In terms of tenderness and blood flow area, there were significant differences between PRE(1) and POST(2) (Figures 4 and 5). Throughout the study period, we found no significant difference in patellar tendon thickness (Figure 6).

Table 1. Evaluation item.

\begin{tabular}{cccccccccccccc}
\hline & \multicolumn{3}{c}{ PRE(1) } & \multicolumn{3}{c}{ POST(1) } & \multicolumn{3}{c}{ PRE(2) } & \multicolumn{3}{c}{ POST(2) } \\
\hline Numeric Rating Scale & 5.36 & \pm & 1.57 & 3.18 & \pm & $1.25^{*}$ & 3.82 & \pm & $1.17^{*}$ & 1.91 & \pm & $1.22^{* t}$ \\
Tenderness (kg) & 1.73 & \pm & 0.98 & 2.45 & \pm & 1.17 & 2.14 & \pm & 0.98 & 3.09 & \pm & $0.97^{*}$ \\
Patella Tendon Thickness (mm) & 0.93 & \pm & 0.26 & 0.93 & \pm & 0.26 & 0.93 & \pm & 0.26 & 0.92 & \pm & 0.26 \\
Blood flow area $\left(\mathrm{cm}^{2}\right)$ & 0.18 & \pm & 0.16 & 0.07 & \pm & 0.08 & 0.13 & \pm & 0.16 & 0.03 & \pm & $0.08^{*}$ \\
\hline
\end{tabular}

Values are mean $\pm \mathrm{SD}^{*}: p<0.05$ PRE(1) ${ }^{\dagger}: p<0.05$ PRE(2).

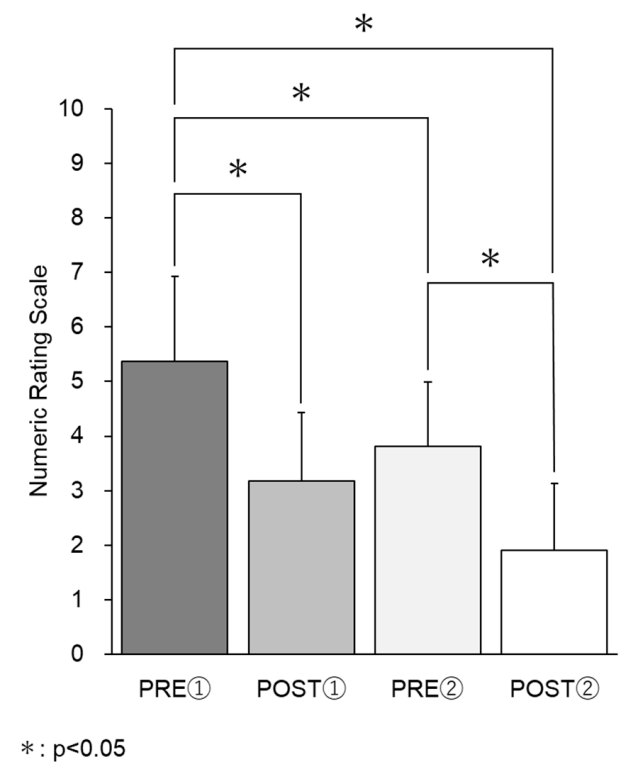

Figure 3. Numeric Rating Scale. 


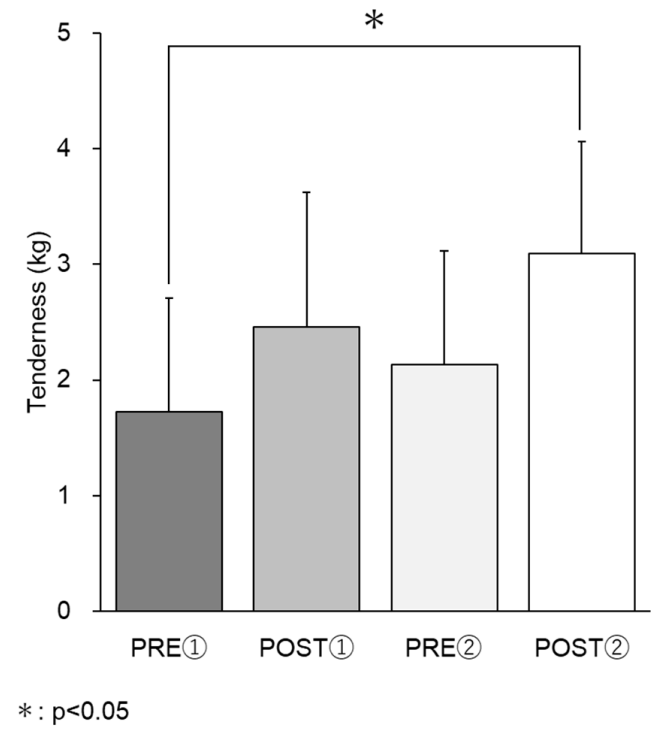

Figure 4. Tenderness.

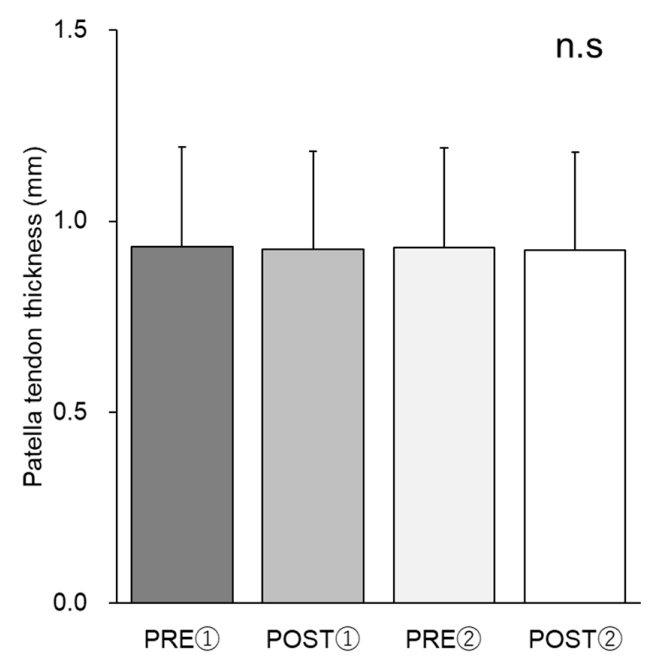

Figure 5. Blood flow area.

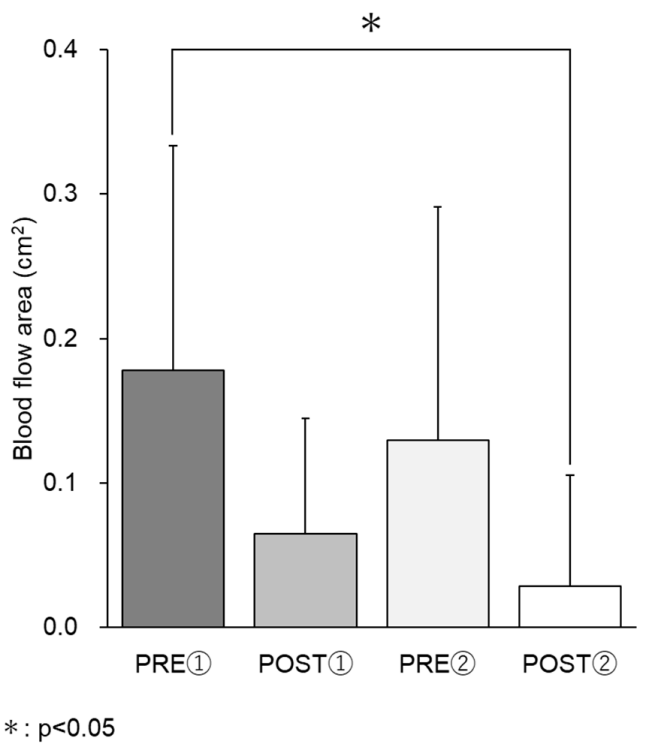

Figure 6. Patella tendon thickness. 


\section{Discussion}

The purpose of this study was to investigate the changes in pain intensity and tendon morphology following f-ESWT using ultrasound imaging to map these changes in patients with patellar tendinopathy. Our study focused on two main questions: immediate pain changes before and after f-ESWT and pain changes two weeks after the first f-ESWT session. On examining the immediate changes in pain before and after the first f-ESWT irradiation, we found no change in patellar tendon thickness but observed a significant decrease in NRS in both the first and second irradiations. Further, although there was no significant difference in tenderness and blood flow area between the first and second irradiation, there was a stark decreasing trend between the two sessions. Previous studies have reported that early pain relief is associated with the destruction of free nerve endings [16,21]. In the present study, pain in the patellar tendon at rest decreased before and after irradiation, suggesting that f-ESWT irradiation may have destroyed nerve endings and had an analgesic effect. Further, the pathogenesis of tendinopathy involves [22,23]. In our participants, this abnormal vascular network tended to decrease after f-ESWT, even though we checked the blood flow area before irradiation. We believe that the destruction of blood vessels by irradiation may have contributed to a decrease in fluid factors, such as neurostimulants, from blood vessels, thereby reducing the pain [2,34]. In a comparative study of pain changes 2 weeks after the first f-ESWT, we found no change in patellar tendon thickness but observed a significant decrease in NRS between PRE(2) and POST(2) compared to PRE(1). We also observed a significant decrease in tenderness and blood flow area in POST (2) as compared to PRE(1). The recommended frequency of irradiation is three sessions at intervals of seven days [35]. In this study, we performed two irradiations performed every 14 days and observed significant improvements in NRS, tenderness and blood flow area in a short period. These results suggest that continuous f-ESWT irradiation over multiple sessions may enhance the pain relief effect and promote the recovery of tissue function [17]. Although this was not significant, we observed increasing trends in NRS, tenderness and blood flow area during the 7 days from POST(1) to PRE(2). Our subjects were college athletes who exercised regularly, and we did not add restrictions on physical activity during the study period. Therefore, they might have engaged in athletic activities during the f-ESWT period, which may have led to an increase in NRS, tenderness and blood flow area. Our results proved the immediate and short-term usefulness of f-ESWT in restoring the condition of an injured patellar tendon. Since the pain relief effect persisted even during exercise in our participants, we believe that f-ESWT is a useful treatment modality that does not require prolonged withdrawal for post-treatment recovery, unlike surgery. In the past, there have been reports that there was no difference in the effects of ESWT with and without irradiation, although subjective pain was reduced [36]. In addition, there may be cases where the tendon is not actually repaired even though it is subjectively improved, thus it is important to combine objective evaluation with subjective evaluation. There are several limitations in our study. First, while evaluating the blood flow area, it was not possible to distinguish between the abnormal vascular network produced during the inflammatory process and the neovascularization produced during the repair process. Second, it can only be tracked up to twice f-ESWT, not long-term. Thirdly, the sample size was small and including a heterogeneous group of unilateral and bilateral patella tendinopathy. Forth, the selection of ultrasound images is made by only one doctor, and the reliability of multiple doctors has not been examined. Given these limitations, further clinical studies on the mechanism of action and efficacy of f-ESWT on patellar tendinopathy are needed.

\section{Conclusions}

The results of this study suggest that f-ESWT provides immediate pain relief in athletes with refractory patellar tendinopathy and that at least two rounds of f-ESWT irradiation in two weeks can promote high pain relief and tissue repair in individuals participating in active sports. 
Author Contributions: Conceptualization: T.M.; methodology: T.M., T.T., T.O. and T.H.; formal analysis and investigation: T.M., T.T., T.O. and T.H.; writing-original draft preparation: T.M.; writing - review and editing: T.H. and T.K.; supervision: T.H. and T.K. All authors have read and agreed to the published version of the manuscript.

Funding: This research received no external funding.

Institutional Review Board Statement: All procedures performed in studies involving human participants were in accordance with the ethical standards of the Ethics Review Committee on Research with Human Subjects of Waseda University and with the 1964 Helsinki declaration and its later amendments or comparable ethical standards.

Informed Consent Statement: Informed consent was obtained from all subjects involved in the study.

Data Availability Statement: The data presented in this study are available on request from the corresponding author.

Acknowledgments: The authors would like to thank all athletes who participated in the measurements.

Conflicts of Interest: The authors declare no conflict of interest.

\section{References}

1. Takahashi, N.; Wada, Y.; Ohtori, S.; Saisu, T.; Moriya, H. Application of shock waves to rat skin decreases calcitonin gene-related peptide immunoreactivity in dorsal root ganglion neurons. Auton. Neurosci. 2003, 107, 81-84. [CrossRef]

2. Hausdorf, J.; Lemmens, M.A.; Heck, K.D.; Grolms, N.; Korr, H.; Kertschanska, S.; Steinbusch, H.W.; Schmitz, C.; Maier, M. Selective loss of unmyelinated nerve fibers after extracorporeal shockwave application to the musculoskeletal system. Neuroscience 2008, 155, 138-144. [CrossRef]

3. van der Worp, H.; Zwerver, J.; Hamstra, M.; van den Akker-Scheek, I.; Diercks, R.L. No difference in effectiveness between focused and radial shockwave therapy for treating patellar tendinopathy: A randomized controlled trial. Knee Surg. Sports Traumatol. Arthrosc. 2014, 22, 2026-2032. [CrossRef]

4. Wheeler, P.C.; Tattersall, C. Novel Interventions for Recalcitrant Achilles Tendinopathy: Benefits Seen Following High-Volume Image-Guided Injection or Extracorporeal Shockwave Therapy-A Prospective Cohort Study. Clin. J. Sport Med. 2020, 30, 14-19. [CrossRef] [PubMed]

5. Maffulli, G.; Padulo, J.; Iuliano, E.; Saxena, A.; Rompe, J.; Maffullii, N. “Extracorporeal shock wave therapy in the treatment of trochanteric bursitis: The ASSERT database". Muscles Ligaments Tendons J. 2019, 8, 444-450. [CrossRef]

6. Oliva, F.; Rugiero, C.; Giai Via, A.; Baldassarri, M.; Bernardi, G.; Biz, C.; Bossa, M.; Buda, R.; Buonocore, D.; Chianca, V.; et al. IS $\mathrm{Mu}$. LT Achilles tendon ruptures guidelines. Muscle Ligaments Tendons J. 2018, 8, 310-363. [CrossRef]

7. Hausdorf, J.; Lemmens, M.A.M.; Kaplan, S.; Marangoz, C.; Milz, S.; Odaci, E.; Korr, H.; Schmitz, C.; Maier, M. Extracorporeal shockwave application to the distal femur of rabbits diminishes the number of neurons immunoreactive for substance P in dorsal root ganglia L5. Brain Res. 2008, 1207, 96-101. [CrossRef]

8. Helland, C.; Bojsen-Møller, J.; Raastad, T.; Seynnes, O.R.; Moltubakk, M.M.; Jakobsen, V.; Visnes, H.; Bahr, R. Mechanical properties of the patellar tendon in elite volleyball players with and without patellar tendinopathy. Br. J. Sports Med. 2013, 47, 862-868. [CrossRef] [PubMed]

9. Kulig, K.; Landel, R.; Chang, Y.-J.; Hannanvash, N.; Reischl, S.F.; Song, P.; Bashford, G.R. Patellar tendon morphology in volleyball athletes with and without patellar tendinopathy. Scand. J. Med. Sci. Sports 2013, 23, 81-88. [CrossRef] [PubMed]

10. Figueroa, D.; Figueroa, F.; Calvo, R. Patellar Tendinopathy: Diagnosis and Treatment. J. Am. Acad. Orthop. Surg. 2016, 24, e184-e192. [CrossRef] [PubMed]

11. Schwartz, A.; Watson, J.N.; Hutchinson, M.R. Patellar Tendinopathy. Sports Health 2015, 7, 415-420. [CrossRef]

12. Cook, J.L.; Khan, K.M.; Kiss, Z.S.; Griffiths, L. Patellar tendinopathy in junior basketball players: A controlled clinical and ultrasonographic study of 268 patellar tendons in players aged 14-18 years. Scand. J. Med. Sci. Sports 2000, 10, 216-220. [CrossRef]

13. Cook, J.L.; Kiss, Z.S.; Khan, K.M.; Purdam, C.R.; Webster, K.E. Anthropometry, physical performance, and ultrasound patellar tendon abnormality in elite junior basketball players: A cross-sectional study. Br. J. Sports Med. 2004, 38, 206-209. [CrossRef]

14. Ackermann, P.W.; Renström, P. Tendinopathy in sport. Sports Health 2012, 4, 193-201. [CrossRef]

15. Han, S.H.; Lee, J.W.; Guyton, G.P.; Parks, B.G.; Courneya, J.-P.; Schon, L.C.J. Leonard Goldner Award 2008. Effect of extracorporeal shock wave therapy on cultured tenocytes. Foot Ankle Int. 2009, 30, 93-98. [CrossRef] [PubMed]

16. Sun, J.; Gao, F.; Wang, Y.; Sun, W.; Jiang, B.; Li, Z. Extracorporeal shock wave therapy is effective in treating chronic plantar fasciitis: A meta-analysis of RCTs. Medicine 2017, 96, e6621. [CrossRef]

17. Gatz, M.; Schweda, S.; Betsch, M.; Dirrichs, T.; de la Fuente, M.; Reinhardt, N.; Quack, V. Line- and Point-Focused Extracorporeal Shock Wave Therapy for Achilles Tendinopathy: A Placebo-Controlled RCT Study. Sports Health 2021, 13, 511-518. [CrossRef] [PubMed] 
18. Wang, C.J.; Ko, J.Y.; Chan, Y.S.; Weng, L.H.; Hsu, S.L. Extracorporeal shockwave for chronic patellar tendinopathy. Am. J. Sports Med. 2007, 35, 972-978. [CrossRef] [PubMed]

19. Furia, J.P.; Rompe, J.D.; Cacchio, A.; Del Buono, A.; Maffulli, N. A single application of low-energy radial extracorporeal shock wave therapy is effective for the management of chronic patellar tendinopathy. Knee Surg. Sports Traumatol. Arthrosc. 2013, 21, 346-350. [CrossRef] [PubMed]

20. Mani-Babu, S.; Morrissey, D.; Waugh, C.; Screen, H.; Barton, C. The effectiveness of extracorporeal shock wave therapy in lower limb tendinopathy: A systematic review. Am. J. Sports Med. 2015, 43, 752-761. [CrossRef]

21. Benjamin, M.; McGonagle, D. Histopathologic changes at 'synovio-entheseal complexes' suggesting a novel mechanism for synovitis in osteoarthritis and spondylarthritis. Arthritis Rheum. 2007, 56, 3601-3609. [CrossRef]

22. Kailash, S.S.K. Shock wave treatment in medicine. J. Biosci. 2005, 30, 269-275. [CrossRef]

23. Orhan, Z.; Alper, M.; Akman, Y.; Yavuz, O.; Yalçiner, A. An experimental study on the application of extracorporeal shock waves in the treatment of tendon injuries: Preliminary report. J. Orthop. Sci. 2001, 6, 566-570. [CrossRef] [PubMed]

24. Wang, C.J.; Huang, H.Y.; Pai, C.H. Shock wave-enhanced neovascularization the tendon-bone junction: An experiment in dogs. J. Foot Ankle Surg. 2002, 41, 16-22. [CrossRef]

25. Blazina, M.E.; Kerlan, R.K.; Jobe, F.W.; Carter, V.S.; Carlson, G.J. Jumper's knee. Orthop. Clin. N. Am. 1973, 4, 665-678. [CrossRef]

26. Roels, J.; Martens, M.; Mulier, J.C.; Burssens, A. Patellar tendinitis (jumper's knee). Am. J. Sports Med. 1978, 6, 362-368. [CrossRef]

27. Lian, O.; Holen, K.J.; Engebretsen, L.; Bahr, R. Relationship between symptoms of jumper's knee and the ultrasound characteristics of the patellar tendon among high level male volleyball players. Scand. J. Med. Sci. Sports 1996, 6, 291-296. [CrossRef] [PubMed]

28. Hardy, A.; Rousseau, R.; Issa, S.P.; Gerometta, A.; Pascal-Moussellard, H.; Granger, B.; Khiami, F. Functional outcomes and return to sports after surgical treatment of insertional Achilles tendinopathy: Surgical approach tailored to the degree of tendon involvement. Orthop. Traumatol. Surg. Res. 2018, 104, 719-723. [CrossRef]

29. Vetrano, M.; Castorina, A.; Vulpiani, M.C.; Baldini, R.; Pavan, A.; Ferretti, A. Platelet-rich plasma versus focused shock waves in the treatment of jumper's knee in athletes. Am. J. Sports Med. 2013, 41, 795-803. [CrossRef]

30. Haefeli, M.; Elfering, A. Pain assessment. Eur. Spine J. 2006, 15 (Suppl. 1), S17-S24. [CrossRef]

31. Kanda, K.; Sakuma, J.; Akimoto, T.; Kawakami, Y.; Suzuki, K. Detection of titin fragments in urine in response to exercise-induced muscle damage. PLoS ONE 2017, 12, e0181623. [CrossRef]

32. Kanda, K.; Sugama, K.; Sakuma, J.; Kawakami, Y.; Suzuki, K. Evaluation of serum leaking enzymes and investigation into new biomarkers for exercise-induced muscle damage. Exerc. Immunol. Rev. 2014, 20, 39-54. [PubMed]

33. Lee, M.; Shin, J.; Kato, T.; Kanda, K.; Oikawa, S.; Sakuma, J.; Sugama, K.; Kawakami, Y.; Suzuki, K.; Akimoto, T. An acute eccentric exercise increases circulating myomesin 3 fragments. J. Physiol. Sci. 2021, 71, 4. [CrossRef]

34. Ohtori, S.; Inoue, G.; Mannoji, C.; Saisu, T.; Takahashi, K.; Mitsuhashi, S.; Wada, Y.; Takahashi, K.; Yamagata, M.; Moriya, H. Shock wave application to rat skin induces degeneration and reinnervation of sensory nerve fibres. Neurosci. Lett. 2001, 315, 57-60. [CrossRef]

35. Schmitz, C.; Császár, N.B.; Milz, S.; Schieker, M.; Maffulli, N.; Rompe, J.D.; Furia, J.P. Efficacy and safety of extracorporeal shock wave therapy for orthopedic conditions: A systematic review on studies listed in the PEDro database. Br. Med. Bull. 2015, 116, 115-138. [CrossRef] [PubMed]

36. Zwerver, J.; Hartgens, F.; Verhagen, E.; van der Worp, H.; van den Akker-Scheek, I.; Diercks, R.L. No effect of extracorporeal shockwave therapy on patellar tendinopathy in jumping athletes during the competitive season: A randomized clinical trial. Am. J. Sports Med. 2011, 39, 1191-1199. [CrossRef] [PubMed] 